Instituto Internacional de Investigación y Desarrollo Tecnológico Educativo INDTEC, C.A.

DOI: https://doi.org/10.29394/Scientific.issn.2542-2987.2020.5.E.6.127-145

OAI-PMH: http://www.indteca.com/ojs/index.php/Revista Scientific/oai

Artículo Original / Original Article

\title{
Consecuencias del consumo de drogas en las Funciones Ejecutivas en adolescentes y jóvenes adultos
}

Autores: Joffre David Agualongo Amangandi Universidad Internacional SEK, UISEK jdagualongo.mpp@uisek.edu.ec

Quito, Ecuador

https://orcid.org/0000-0001-6822-6290

Diana Isabel Robalino Robayo Universidad Internacional SEK, UISEK diana.robalino@uisek.edu.ec

Quito, Ecuador

https://orcid.org/0000-0001-7274-7902

\section{Resumen}

El consumo prolongado de diferentes tipos de drogas trae como consecuencia, entre algunas, el deterioro de diversas capacidades cognitivas, entre las que resaltan por su importancia clínica, las Funciones Ejecutivas. Estas funciones neuropsicológicas son consideradas como aquellas que permiten al ser humano prever, organizar información, razonar, generar metas y planes, inhibir conductas, entre otras, para construir estrategias tanto cognitivas, conductuales y emocionales necesarias para resolver problemas. El presente artículo tiene como objetivo principal conocer las habilidades ejecutivas alteradas debido al consumo de drogas en las personas que asisten al Área Ambulatorio Intensiva del Centro de Salud de ciudad de la Latacunga, Ecuador. Se empleó una metodología cuantitativa de tipo descriptivo, con una muestra de 20 personas. El instrumento psicométrico aplicado fue la Batería Neuropsicológica de Funciones Ejecutivas y Lóbulos Frontales (BANFE-1). Dentro de los resultados, el $50 \%$ de individuos presentó alteración severa en el funcionamiento global de las funciones ejecutivas. Específicamente, el $40 \%$ de estos, evidenciaron alteración severa del córtex dorsolateral, donde las habilidades ejecutivas que fueron afectadas por el consumo de drogas son: control inhibitorio, flexibilidad cognitiva, memoria operativa o de trabajo, control de impulsos o autocontrol, planificación y organización secuencial.

Palabras clave: adicción; evaluación; psicología clínica.

Cómo citar este artículo:

Agualongo, J., \& Robalino, D. (2020). Consecuencias del consumo de drogas en las Funciones Ejecutivas en adolescentes y jóvenes adultos. Revista Scientific, 5(Ed. Esp.), 127-145, e-ISSN: 2542-2987. Recuperado de: https://doi.org/10.29394/Scientific.issn.2542-2987.2020.5.E.6.127-145

Fecha de Recepción:

07-04-2020
Fecha de Aceptación:

24-06-2020
Fecha de Publicación:

05-08-2020 
OAI-PMH: http://www.indteca.com/ojs/index.php/Revista_Scientific/oai

Artículo Original / Original Article

\section{Consequences of drug use on executive functions in adolescents and young adults}

\begin{abstract}
The prolonged use of different types of drugs results in, among some, the deterioration of various cognitive capacities, among which the Executive Functions stand out for their clinical importance. These neuropsychological functions are considered as those that allow the human being to foresee, organize information, reason, generate goals and plans, inhibit behaviors, among others, to build both cognitive, behavioral and emotional strategies necessary to solve problems. The main objective of this article is to find out about the executive abilities altered due to drug consumption in people who attend the Intensive Outpatient Area of the Health Center of the city of Latacunga, Ecuador. A descriptive quantitative methodology was used, with a sample of 20 people. The applied psychometric instrument was the Neuropsychological Battery of Executive Functions and Frontal Lobes (BANFE-1). Within the results, $50 \%$ of individuals presented severe alteration in the global functioning of executive functions. Specifically, $40 \%$ of these evidenced severe alteration of the dorsolateral cortex, where the executive skills that were affected by drug use are: inhibitory control, cognitive flexibility, working or working memory, impulse control or self-control, planning and organization sequential.
\end{abstract}

Keywords: addiction; evaluation; clinical psychology.
How to cite this article:

Agualongo, J., \& Robalino, D. (2020). Consequences of drug use on executive functions in adolescents and young adults. Revista Scientific, 5(Ed. Esp.), 127-145, e-ISSN: 2542-2987. Recovered from: https://doi.org/10.29394/Scientific.issn.2542-2987.2020.5.E.6.127-145
Date Received:

07-04-2020
Date Acceptance:

24-06-2020
Date Publication:

05-08-2020 


\section{Introducción}

En el Ecuador, son escasos, casi nulos los estudios validados instrumentalmente sobre los efectos de las drogas en el funcionamiento cognitivo, más aún en lo que refiere a funciones ejecutivas, sin embargo, existen investigaciones de otros países como Estados Unidos, España, Colombia, etc., que evidencian que el consumo de drogas tiene incidencia a nivel de funcionamiento neuropsicológico.

Dentro de esta investigación, se ha tomado como referencia teórica primordial a las ciencias neuropsicológicas, encargadas del estudio de la relación entre la conducta y cerebro, quienes toman en cuenta las secuelas que tanto a nivel cognitivo y comportamental puede provocar una lesión en este órgano. Así, se puede definir Neuropsicología o Neurociencia Cognitiva, según Portellano (2005): es como el estudio de las capacidades mentales superiores, regularmente llamadas funciones cognitivas.

Más a profundidad, la Neuropsicología de orientación psicológica o Neuropsicología cognitiva, paralelamente Manga y Ramos (2001): estudian los procesos psicológicos, principalmente cognitivos, en cuadros clínicos diversos tales como lesiones cerebrales, trastornos mentales, etc. Dentro de esta orientación, los test psicométricos se utilizan como instrumentos para obtener resultados, teniendo en cuenta que la observación clínica por sí sola no es suficiente para establecer resultados o conclusiones efectivas.

Desde este punto, es necesario definir qué son las funciones ejecutivas e irlas diferenciando de las funciones cognitivas. De acuerdo con Portellano, Martínez y Zumárraga (2009): las funciones ejecutivas son un "conjunto de capacidades que nos permiten transformar nuestros pensamientos en decisiones, planes y acciones, consiguiendo un mejor grado de adaptación a nuestro entorno" (pág. 20). Este término inicialmente fue nombrado por Lezak (1982), citado por Tirapu-Ustárroz y Muñoz-Céspedes (2005): para hacer referencia a una variedad de capacidades relacionadas con la planificación, 
organización y ejecución de acciones.

Para diferenciar entre función ejecutiva y función cognitiva, este último autor, indica que las funciones cognitivas generales son procesos mentales que facilitan al individuo recibir, procesar y transformar información, para poder comprender e interactuar con el medio que le rodea. Frente a ello, Lezak, Howieson y Loring (2004): expone que la función ejecutiva es la capacidad que lleva a una persona a ser independiente, propositiva y autorregulada a nivel comportamental, es decir, permite a la persona saber cómo, cuándo y para qué se lleva a cabo una acción, siendo esta, parte de las funciones cognitivas superiores.

El interés por investigar las alteraciones en las capacidades cognitivas, como las funciones ejecutivas se explica, entre otras razones, porque se ha demostrado que el consumo excesivo y prolongado de drogas ocasiona cambios neuroanatómicos y funcionales. Estos cambios para Ruiz y Pérez (2014): pueden mantenerse en zonas cerebrales que están involucradas en distintas funciones cognitivas, tales como la motivación, toma de decisiones, la atención, la memoria entre otras habilidades.

Mencionando a Muñoz y Tirapú (2001): dichas habilidades han sido razón de valoración en diversas investigaciones en el campo científico, con el propósito de establecer perfiles clínicos de desenvolvimiento cognitivo, emocional y conductual. Indica Lezak (1983), citado por Rosselli, Matute y Jurado (2008): que es la disfunción de las funciones ejecutivas la que puede delimitar las aptitudes de la persona para conservar una vida autónoma y provechosa, incluso si otras destrezas cognoscitivas se encuentren íntegras, de acuerdo con Ardila y Rosselli (2007): estas involucran habilidades como la facultad para discernir información no pertinente, el control de las acciones o encaminadas hacia una meta, la habilidad de prever e inhibir conductas y la flexibilidad cognitiva.

Otros autores como Tirapu-Ustárroz, García, Ríos-Lago y Ardila (2012): 
hacen referencia a las funciones ejecutivas como las destrezas que tenemos para resolver conflictos y hallar soluciones, para la cual se necesita llevar a cabo una serie de anticipaciones de los posibles efectos a los que podemos llegar con cada una de las estrategias trazadas.

Así, Corral, Rodríguez y Cadaveira (2002): verifican una afectación de las funciones implicadas en la resolución de problemas y situaciones novedosas entre los individuos que consumen alcohol, y que a la vez abarcan características como la organización, la abstracción, la contención de respuestas irrelevantes, la flexibilidad mental, las destrezas cognitivas.

Los programas de tratamiento para personas que consumen o han consumido algún tipo de droga se abordan dentro de un contexto de contención y prevención de situaciones de riesgo para los pacientes. Este principio se verifica tanto en el servicio privado como en el servicio estatal, regido por el Ministerio de Salud Pública del Ecuador, a través de los Centros Especializados de Tratamiento a Personas con Consumo Problemático de Alcohol y otras Drogas (CETAD) y el Servicio Ambulatorio Intensivo (SAI).

El Ministerio de Salud Pública (2015): presenta que la revisión de los lineamientos generales de la intervención terapéutica respalda la autonomía de la persona, en general, y sugiere la reconstrucción de su proyecto de vida en particular. Sin embargo, no existen intervenciones focalizadas en el daño neuronal de áreas cerebrales específicas y, por ende, en la rehabilitación de funciones cognitivas, lo que denota la escasa relevancia que adquiere el abordaje de estas capacidades en el contexto general de los programas de tratamiento.

Así lo afirman Muñoz-Céspedes y Tirapu-Ustárroz (2004): al considerar que los individuos con quebranto en el funcionamiento ejecutivo denotan severas complicaciones para organizar y operar eficientemente su conducta, evidenciándose en comportamientos que carecen de coherencia e interfiere en una adecuada generalización de los aprendizajes, y por ello afirman que 
es habitual que las alteraciones ejecutivas compongan un fin fundamental de cualquier plan de restitución neuropsicológica, dado que este tipo de déficit es responsable de varios de los impedimentos más significativos que obstaculizan a estas personas hacer frente a situaciones nuevas $e$ inesperadas.

De los trabajos realizados en California por Fein, Bachman, Fisher y Davenport (1990) citados por Landa, Fernández-Montalvo y Tirapu (2004): aseveran que el déficit cognitivo en alcohólicos es extenso y frecuente, y demuestran que, entre las habilidades más alteradas en esos primeros instantes, se encuentra la capacidad para realizar nuevos aprendizajes. Sin embargo, así mismo posibilitan que estos pacientes pueden recuperar niveles premórbidos tras un ciclo extendido de abstinencia.

Frente a esto, Madoz-Gúrpide y Ochoa-Mangado (2012): en sus investigaciones realizada en España sobre alternaciones de funciones cognitivas y ejecutivas en pacientes dependientes de cocaína, confirman un predominio claro de deterioro en las funciones ejecutivas, en el dominio de impulsos y abstención de estímulos insignificantes, además resaltan que dichas alteraciones facilitan mostrar en parte el fracaso terapéutico que con reiteración se da en este grupo de personas e indica la necesidad de adecuar los tratamientos al desenvolvimiento cognitivo real del sujeto.

Estudios realizados en Colombia por Mariño, Castro y Torrado (2012): sobre las alteraciones en funciones ejecutivas en policonsumidores de sustancias psicoactivas, establecen que en las áreas frontales, las zonas con mayor nivel de alteración cognitiva son la corteza motora premotora encargada de la capacidad de planear, la corteza frontomedial, responsable de la atención; y por último, la corteza prefrontal dorso-lateral, responsable de acciones como la memoria, la fluidez y la flexibilidad mental.

En una investigación elaborada en Ecuador por Poveda, Mora y Naranjo (2017): sobre aspectos neuropsicológicos y sociales de la drogodependencia, 
exponen evidencias que se relacionan a alteración significativa en la región orbito-medial del lóbulo prefrontal del cerebro en consumidores de droga, lo que daría a entender que existe dificultad en el desarrollo del control inhibitorio durante una actividad. Este resultado a su vez, se relacionaría con la falta de retroalimentación ante un error durante una tarea determinada y la pertinente corrección de la misma para evitar un resultado inadecuado.

Por tanto, para consolidar lo concerniente a las funciones ejecutivas, se puede manifestar que todos los estudios revisados tienen convergencia, e indican que una afectación a este nivel dificulta varias de las habilidades del ser humano. No obstante, desde nuestra práctica y experiencia profesional dentro del ámbito clínico, podemos mencionar que no se encuentra formalizado un proceso de evaluación y diagnóstico neuropsicológico básico que incluya el análisis del funcionamiento de una serie de procesos cognitivos y conductuales afectados por el consumo prolongado de drogas.

Tampoco se encuentra especificada la utilización de pruebas neuropsicológicas válidas que evidencien de manera más específica disfunciones cerebrales que aportarían a un mejoramiento en el pronóstico de la recuperación, un plan de rehabilitación adecuado, así como también aportaría a la adherencia, retención y disminución de recaídas del consumo. Estas valoraciones neuropsicológicas realizadas están enfocadas precisamente a conocer la afectación en las áreas del córtex prefrontal y cómo esta puede repercutir en las diferentes áreas de la vida diaria como son, área familiar, social, económica, académica o laboral y personal.

En este punto es necesario recalcar que la falta de investigación sobre las intervenciones neuropsicológicas en el consumo de drogas, trae como consecuencia que los profesionales que atienden estos casos no lo consideren lo suficientemente importante ni siquiera como parte de la evaluación psicológica inicial y peor aún en la rehabilitación cognitiva dentro del tratamiento que plantean y que aplican, el mismo que, interpretando a Mateer 
(2003), citado por Arango (2006): debería ser un proceso completo en donde se utilice adecuadamente procedimientos y técnicas que permitan a las personas con déficit cognitivos retomar sus actividades cotidianas.

En tal virtud, el objetivo de esta investigación es determinar cuáles son las habilidades ejecutivas, dentro del funcionamiento ejecutivo global, mayormente alteradas por el consumo prolongado de alcohol y otras drogas a partir del índice de alteración de cada una de las tres áreas prefrontales evaluadas. De esta manera, los resultados expuestos pueden ser tomados como referencia para elaborar y aplicar diferentes estrategias de rehabilitación cognitiva individual y grupal que mejore el desenvolvimiento conductual, social, emocional y familiar de este grupo poblacional, las cuales deberían utilizarse junto con el trabajo en equipo de otras áreas de especialización médica.

\section{Metodología (Materiales y métodos)}

Estudio de tipo transversal donde se utilizó una metodología cuantitativa de tipo descriptivo, ya que, a partir de los resultados de la aplicación de la prueba batería Neuropsicológica de Funciones Ejecutivas y Lóbulos Frontales BANFE-1, se determinó las habilidades ejecutivas mayormente afectadas en el desempeño cognitivo de consumidores de drogas. Se aplicó el método estadístico descriptivo simple, empleando el software de estadística IBM SPSS Statistics Base 22.0 para conocer las frecuencias de ocurrencia de cada diagnóstico, así como los porcentajes de la población según los niveles de afectación, lo que llevó a inferir objetivamente que el consumo prolongado de drogas afecta el funcionamiento del lóbulo prefrontal del cerebro.

Se tomó un muestreo no probabilístico durante dos meses a 20 individuos que asistían al Servicio Ambulatorio Intensivo (SAI) del centro de salud tipo "C" de Latacunga, sin embargo, se debe indicar que inicialmente la población a ser evaluada era de 27 participantes, pero debido a los criterios de inclusión y exclusión, esta muestra se redujo a la cantidad antes indicada. 
2.1. Criterios de inclusión: individuos que hayan consumido cualquier tipo de droga en un tiempo no menor a un año y de manera continua, que tengan edades entre 15 y 25 años, de distintos niveles de escolaridad, además que se encuentren en proceso de recuperación y asistan al SAl.

2.2. Criterios de exclusión: Individuos menores de 15 o mayores de 25 años, que presenten algún diagnóstico de trastorno mental no asociado al consumo de drogas, que no se encuentren en proceso de recuperación, que presenten un consumo ocasional y que esta conducta no es problemática.

Se empleó como material principal el BANFE-1, que tiene como objetivo evaluar el desarrollo de las funciones ejecutivas a través de 15 procesos. Éstos se agrupan en tres áreas cerebrales: orbitomedial, prefrontal anterior y dorsolateral.

La batería utilizada se seleccionó en función de su validez neuropsicológica principalmente. Su confiabilidad se encuentra respaldada por miembros eminentes de la comunidad científica internacional y cuenta con suficiente soporte en la literatura científica.

\section{Resultados (análisis e interpretación de los resultados)}

En la totalización general del desempeño poblacional de las habilidades cognitivas correspondientes a las funciones ejecutivas, en cuanto al rendimiento general, el $40 \%$ de la población estudiada presenta alteración leve y un $50 \%$ de la población manifiesta alteración severa. Lo que quiere decir que solo el $10 \%$ de esta población no tuvo alteración en el lóbulo prefrontal del cerebro correspondiente a funcionamiento ejecutivo global.

A partir de la lectura del análisis descriptivo de los resultados de la tabulación de la hoja resumen BANFE-1, se procedió a realizar un análisis específico de las subpruebas contempladas en el acápite correspondiente a la batería de funciones ejecutivas, en donde se determinó las tareas con menor desempeño puntuadas por debajo de la media 2.5 teniendo en cuenta que la 
puntuación máxima fue de 5 puntos. De esta manera, los resultados más importantes que se evidenciaron fueron:

3.1. Test de Stroop: evalúa la habilidad atencional para poder inhibir estímulos visuales y verbales no importantes y necesarios para el desarrollo de una tarea. Los resultados fueron: errores tipo Stroop "A" el resultado fue 2 puntos y errores de tiempo Stroop "A y B" obtienen 2,2.

3.2. Clasificación de cartas: evalúa flexibilidad cognitiva, es decir, la capacidad abstracta para cambiar de estrategia en la resolución de un problema, evitando el mantenimiento de intentos fallidos para lograr el cometido. Los resultados fueron: 2,3 en el apartado errores de mantenimiento.

3.3. Señalamiento autodirigido: evalúa la capacidad para retener, por una pequeña cantidad de tiempo, información de tipo visoespacial al señalar una serie de figuras previamente indicadas. Los resultados fueron: 2,05 en el tiempo que se demoraron en realizar la tarea.

3.4. Memoria visoespacial: evalúa la destreza para conservar, de manera ordenada y secuencial, información visual y resolver problemas de manera inmediata. Los resultados fueron: 2,05 en errores de orden.

3.5. Laberintos: Evalúa la habilidad para planear y organizar una conducta, desde información visual que ha obtenido con anterioridad, lo que le permite ser rápido para cumplir un objetivo. Los resultados fueron: 2,06 en planeación y 1,85 en tiempo.

3.6. Torres de Hanói: evalúa la capacidad resolver un problema de manera ordenada por medio de la planificación secuencial de movimientos. Los resultados fueron: 2,45 en el índice de movimientos de tres fichas; 2 puntos en tiempo utilizado; y 2,1 en el índice de movimientos de cuatro fichas.

A partir de la aplicación de la batería BANFE-1, se pudo determinar, que el desempeño de las funciones ejecutivas se encuentra afectada de manera global en más de la mitad de los consumidores prolongados de droga 
evaluados, como se presenta en la tabla 1. De ellos, $44 \%$ presentan alteración leve, y $56 \%$ alteración severa. Apenas el $10 \%$ de la muestra total, denota funcionamiento normal de las capacidades ejecutivas.

Tabla 1. Diagnóstico de la Batería Neuropsicológica BANFE-1 en el funcionamiento ejecutivo (F: Frecuencia de ocurrencia, \%: Porcentaje real, \% Acu.: Porcentaje acumulado).

\begin{tabular}{|l|c|c|c|c|c|c|c|c|c|c|c|c|}
\cline { 2 - 13 } \multicolumn{1}{c|}{} & \multicolumn{3}{c|}{ Orbito-medial } & \multicolumn{3}{c|}{ Dorsolateral } & \multicolumn{4}{c|}{$\begin{array}{c}\text { Prefrontal } \\
\text { Anterior }\end{array}$} & \multicolumn{3}{c|}{$\begin{array}{c}\text { Funciones } \\
\text { Ejecutivas }\end{array}$} \\
\cline { 2 - 13 } & $\mathrm{F}$ & $\%$ & $\%$ Acu. & $\mathrm{F}$ & $\%$ & $\%$ Acu. & $\mathrm{F}$ & $\%$ & $\%$ Acu. & $\mathrm{F}$ & $\%$ & $\%$ Acu. \\
\hline $\begin{array}{l}\text { Normal } \\
\text { alto }\end{array}$ & 0 & $0 \%$ & $0 \%$ & 0 & $0 \%$ & $0 \%$ & 0 & $0 \%$ & $0 \%$ & 0 & $0 \%$ & $0 \%$ \\
\hline Normal & 3 & $15 \%$ & $15 \%$ & 7 & $35 \%$ & $35 \%$ & 8 & $40 \%$ & $40 \%$ & 2 & $10 \%$ & $10 \%$ \\
\hline $\begin{array}{l}\text { Alteración } \\
\text { leve }\end{array}$ & 10 & $50 \%$ & $65 \%$ & 5 & $25 \%$ & $60 \%$ & 10 & $50 \%$ & $90 \%$ & 8 & $40 \%$ & $50 \%$ \\
\hline $\begin{array}{l}\text { Alteración } \\
\text { severa }\end{array}$ & 7 & $35 \%$ & $100 \%$ & 8 & $40 \%$ & $100 \%$ & 2 & $10 \%$ & $100 \%$ & 10 & $50 \%$ & $100 \%$ \\
\hline Total & 20 & \multicolumn{2}{|c|}{$100 \%$} & 20 & \multicolumn{2}{c|}{$100 \%$} & 20 & $100 \%$ & 20 & $100 \%$ \\
\hline
\end{tabular}

Fuente: Los Autores (2020).

En la misma tabla, se puede evidenciar que de todos los evaluados, el $40 \%$ presenta alteración severa del córtex dorsolateral del área prefrontal del cerebro, zona encargada de habilidades cognitivas y emocionales como la atención, memoria operativa, planificación, flexibilidad cognitiva, modulación de la conducta, motivación, autocontrol, etc. Así también, se observa que el $35 \%$ de los sujetos evaluados presentan alteración moderada en el córtex orbito-medial, área que involucra capacidades cognitivas como procesamiento y regulación emocional, autoconciencia, sentido ético, toma de decisiones, entre otras.

Por otro lado, se puede observar que el área menos afectada es el prefrontal anterior, no por ello también se observan dificultades en habilidades que se procesan en esta estructura como la memoria visoespacial y planificación de tipo visual.

Las habilidades ejecutivas que se evidencian alteradas de manera 
específicas se encuentran descritas en la tabla 2. En este grupo de personas investigadas, se puede determinar, cada una de las pruebas vinculadas a cada área frontal. Se evidencia así que las habilidades más afectadas en el área orbito frontal con un rendimiento medio de 2,18 (bajo la media) están relacionadas a dificultades para mantener la atención, irritabilidad, conducta desinhibida, alteración en el control inhibitorio sin tomar conciencia de la consecuencia de los actos, entre otros.

Tabla 2. Análisis descriptivo de las subpruebas de la batería de funciones ejecutivas.

\begin{tabular}{|l|l|l|l|l|l|}
\hline \multicolumn{7}{|c|}{ Estadísticos descriptivos } \\
\hline \multicolumn{1}{|c|}{ Orbitomedial } & $\mathbf{N}$ & Mínimo & Máximo & Media & Desviación estándar \\
\hline Stroop forma "A". Errores tipo Stroop & 20 & 1,00 & 5,00 & 2,0000 &, 85840 \\
\hline Stroop forma "A". Tiempo & 20 & 1,00 & 3,00 & 2,2000 &, 95145 \\
\hline Stroop forma "B". Tiempo de cartas. Errores de & 20 & 1,00 & 3,00 & 2,2000 &, 89443 \\
\hline $\begin{array}{l}\text { Clasificación de } \\
\text { mantenimiento }\end{array}$ & 20 & 1,00 & 7,00 & 2,3000 & 1,34164 \\
\hline \multicolumn{1}{|c|}{ Prefrontal Anterior } & $\mathbf{N}$ & Mínimo & Máximo & Media & Desviación estándar \\
\hline Señalamiento autodirigido. Tiempo & 20 & 1,00 & 3,00 & 2,0500 &, 99868 \\
\hline $\begin{array}{l}\text { Memoria Visoespacial. Errores de } \\
\text { orden }\end{array}$ & 20 & 1,00 & 4,00 & 2,0500 & 1,09904 \\
\hline Dorsolateral. Funciones Ejecutivas & $\mathbf{N}$ & Mínimo & Máximo & Media & Desviación estándar \\
\hline Laberinto. Planeación & 20 & 2,00 & 5,00 & 2,0600 &, 85224 \\
\hline Laberintos. Tiempo & 20 & 1,00 & 3,00 & 1,8500 &, 93330 \\
\hline Torre de Hanoi 3 fichas. Movimientos & 20 & 2,00 & 5,00 & 2,4500 & 1,10501 \\
\hline Torre de Hanoi 3 fichas. Tiempo & 20 & 1,00 & 3,00 & 2,0000 & 1,02598 \\
\hline Torre de Hanoi 4 fichas. Tiempo & 20 & 1,00 & 3,00 & 2,1000 &, 96791 \\
\hline
\end{tabular}

Fuente: Los Autores (2020).

En relación, al área dorsolateral, donde el promedio es de 2,09 (bajo la media), da cuenta de alteración en capacidades como la memoria de trabajo, flexibilidad cognitiva, planificación y razonamiento abstracto. Finalmente, el área prefrontal anterior, con un rendimiento promedio 2,05 (bajo la media), se evidencian dificultades en procesos de planificación visoespacial, organización secuencial, como también habilidades superiores como metacognición y automonitoreo. 


\section{Conclusiones}

Desde la perspectiva neuropsicológica que ha guiado este trabajo de investigación, se puede manifestar que el consumo de drogas provoca afecciones funcionales en las capacidades ejecutivas del cerebro, lo cual no les permite adaptarse al desenvolvimiento normal en cada uno de los ambientes en los que se desarrollan.

Las personas que han consumido drogas por más de un año, necesariamente deberán ser evaluadas a nivel neuropsicológico, de manera que se pueda conocer el grado de afectación cognitiva, específicamente de su funcionamiento ejecutivo, el cual está influyendo para mostrar dificultades en la readaptación a sus rutinas

Es importante agregar, que en el tratamiento de las personas que han tenido un consumo prolongado de cualquier tipo de drogas debe contener por lo menos un módulo dedicado a la rehabilitación neuropsicológica encaminada a la disminución de los déficits ejecutivos producto de la conducta adictiva, ya que como se expone durante el desarrollo del artículo, en los lineamientos de intervención tanto en la parte privada y pública no involucran dentro de sus servicios programas encaminados a restaurar, compensar o sustituir las capacidades cognitivas.

Lo que se ha expuesto anteriormente, da cuenta de la importancia del trabajo con otros profesionales cuando se presentan este tipo de problemáticas, pues el protocolo de atención se limitan a que el psicólogo clínico evalúe aspectos emocionales y de personalidad, sin tomar necesariamente en cuenta, teorías de funcionamiento neuropsicológico que junto con su evaluación e interpretación son básicas para construir un plan de rehabilitación completa y pertinente que permita a la persona alcanzar un grado de reinserción social adecuado, es muy probable que estos resultados también sean una de las causas para que los consumidores de drogas no desarrollen fácilmente una adhesión a un tratamiento, sin embargo este último 
punto sería objetivo de una nueva investigación.

Los estudios de esta población dentro del área neuropsicológica recién ha empezado, es necesario seguir planteando hipótesis que lleven a la aplicación de varios test normalizados no solo en adultos, sino también en adolescentes, e inclusos niños que tempranamente empiezan a consumir drogas, que permitan evidenciar resultados que comparen no solo las diferencias entre edad, sino de las consecuencias neuropsicológicas entre hombres y mujeres, consecuencias específicas en relación a diferentes drogas, entre otras, para que de esa manera se pueda plantear cada vez mejores y completos planes de rehabilitación que puedan ser aplicado no solo a nivel privado, sino y sobre todo a nivel de atención pública.

Finalmente, el análisis de tipo estadístico realizado permitió concluir que las consecuencias negativas del consumo de drogas, en jóvenes entre 15 a 25 años, en el funcionamiento del lóbulo frontal afecta evidentemente el rendimiento ejecutivo en estas personas. Así, la alteración de los procesos cognitivos ejecutivos, están siempre vinculadas a habilidades que le permiten a la persona desarrollar una vida independiente, autónoma y socialmente adaptada, en este caso, especialmente en el área orbito-medial encargada de procesar información vinculada a la regulación emocional, autoconciencia, toma de decisiones, y sentido ético, mientras que las capacidades como la planificación visoespacial y superiores como la metacognición son las menos afectadas.

\section{Referencias}

Arango, J. (2006). Rehabilitación neuropsicológica. ISBN: 970-729-234-2, ISBN: 978-607-448-134. México, D.F.: Editorial El Manual Moderno, S.A. de C.V.

Ardila, A., \& Rosselli, M. (2007). Neuropsicología Clínica. ISBN 10: 970-729279-2, ISBN 13: 978-970-729-279-6. México, D.F.: Editorial El Manual 
Moderno, S.A. de C.V.

Corral, M., Rodríguez, S., \& Cadaveira, F. (2002). Perfil neuropsicológico de alcohólicos con alta densidad familiar de alcoholismo tras abstinencia prolongada. Revista española de drogodependencias, (2), 148-158, e-ISSN: 0213-7615. Recuperado de:

https://dialnet.unirioja.es/servlet/articulo?codigo $=305649$

Landa, N., Fernández-Montalvo, J., \& Tirapu, J. (2004). Alteraciones neuropsicológicas en el alcoholismo: una revisión sobre la afectación de la memoria y las funciones ejecutivas. Adicciones, 16(1), 41-52, e-ISSN: 0214-4840. Recuperado de:

https://doi.org/10.20882/adicciones.417

Lezak, M., Howieson, D., \& Loring, D. (2004). Neuropsychological Assessment. 4ta Edition. New York, United States: Oxford University Press.

Madoz-Gúrpide, A., \& Ochoa-Mangado, E. (2012). Alteraciones de funciones cognitivas y ejecutivas en pacientes dependientes de cocaína: estudio de casos y controles. Revista de Neurología, 54(4), 199-208, e-ISSN: 1576-6578. Recuperado de:

https://doi.org/10.33588/rn.5404.2011174

Manga, D., \& Ramos, F. (2001). Evaluación de los síndromes neuropsicológicos infantiles. Revista de Neurología, 32(7), 664-675, e-ISSN: 1576-6578. Recuperado de:

https://doi.org/10.33588/rn.3207.2000176

Mariño, N., Castro, J., \& Torrado, J. (2012). Funcionamiento ejecutivo en policonsumidores de sustancias psicoactivas. Revista de Psicología Universidad de Antioquia, 4(2), 49-64, e-ISSN: 2256-1102. Recuperado de:

https://revistas.udea.edu.co/index.php/psicologia/article/view/15480

Ministerio de Salud Pública (2015). Modelo de atención integral residencial 
para el consumo problemático de alcohol y otras drogas, en establecimientos de salud de tercer nivel del Ministerio de Salud Pública. Quito, Ecuador: Subsecretaria Nacional de Provisión de Servicios, Proyecto Creación e Implementación de Servicios de la Red de Salud Mental Comunitaria y Centros Estatales de Recuperación de Adicciones.

Muñoz, J., \& Tirapú, J. (2001). Rehabilitación Neuropsicológica. Madrid, España: Editorial Síntesis.

Muñoz-Céspedes, J., \& Tirapu-Ustárroz, J. (2004). Rehabilitación de las funciones ejecutivas. Revista de Neurología, 38(7), 656-663, e-ISSN: 1576-6578. Recuperado de: https://doi.org/10.33588/rn.3807.2003411 Portellano, J. (2005). Introducción a la neuropsicología. ISBN: 9788448198213. Madrid, España: McGraw-Hill.

Portellano, J., Martínez, R., \& Zumárraga, L. (2009). ENFEN: Evaluación Neuropsicológica de las funciones ejecutivas en niños. ISBN: 97884-15262-32-9. Madrid, España: TEA ediciones.

Poveda, S., Mora, A., \& Naranjo, T. (2017). Aspectos neuropsicológicos y sociales de la drogodependencia en Ecuador. I Congreso de: Ciencia, Sociedad e Investigación Universitaria, 1-22, e-ISSN: 2550679X. Recuperado de:

https://repositorio.pucesa.edu.ec/handle/123456789/2073

Rosselli, M., Matute, E., \& Jurado, M. (2008). Las Funciones Ejecutivas a través de la Vida. Revista Neuropsicología, Neuropsiquiatría y Neurociencias, 8(1), 23-46, e-ISSN: 0124-1265. Recuperado de: https://dialnet.unirioja.es/servlet/articulo?codigo $=3987451$

Ruiz, J., \& Pérez, E. (2014). Neuropsicología de la adicción. ISBN: 9788498357745. Buenos Aires, Argentina: Editorial Médica Panamericana.

Tirapu-Ustárroz, J., \& Muñoz-Céspedes, J.M. (2005). Memoria y funciones 
ejecutivas. Revista de Neurología, 41(8), 475-484, e-ISSN: 1576-6578. Recuperado de: https://doi.org/10.33588/rn.4108.2005240

Tirapu-Ustárroz, J., García, A., Ríos-Lago, M., \& Ardila, A. (2012). Neuropsicología de la corteza prefrontal y las funciones ejecutivas. ISBN: 978-84-92931-13-2. España: Editorial Viguera. 


\section{Joffre David Agualongo Amangandi}

e-mail: jdagualongo.mpp@uisek.edu.ec

Nacido en Guaranda, Ecuador, el 24 de abril del año 1990.

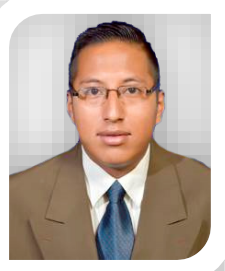

Psicólogo Clínico, graduado en la Universidad Nacional de Chimborazo (UNACH) de la ciudad de Riobamba; Magister en Psicología con Mención en Psicoterapia por la Universidad Internacional SEK (UISEK); Analista del Departamento de Consejería Estudiantil (DECE) en la Unidad Educativa del Milenio en Salinas de Guaranda; Actualmente Psicólogo Clínico y Psicoterapeuta del Centro de Atención Psicológica (SAMENT). 


\section{Diana Isabel Robalino Robayo \\ e-mail: diana.robalino@uisek.edu.ec}

Nacida en Quito, Ecuador, el 29 de diciembre del año

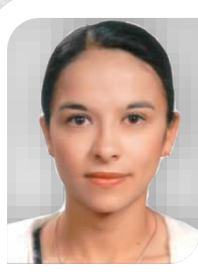
1984. Psicóloga Clínica de la Pontificia Universidad Católica del Ecuador (PUCE); Licenciada en Educación Infantil; Magíster en Neuropsicología Infantil en la Universidad Central del Ecuador (UCE); y en Psicología Clínica y de la Salud en el Instituto Superior de Estudios Psicológicos (ISEP) de Barcelona, España; Diplomado en Estrategias de Intervención Temprana en Salud Mental en la Universidad del Desarrollo (UDD) en Santiago de Chile; Especializada en evaluación y terapia emocional y neuropsicológica de niños y adolescentes; Docente universitaria desde el año 2013 en el área de Psicología, Educación y Psicopedagogía.

El contenido de este manuscrito se difunde bajo una Licencia de Creative Commons ReconocimientoNoComercial-Compartirlgual 4.0 Internacional 